MIFP-05-35

\title{
A Review of Distributions on the String Landscape
}

\author{
Jason Kumar \\ Department of Physics \\ Texas A $B M$ University \\ College Station, TX 77840, USA
}

\begin{abstract}
We review some basic flux vacua counting techniques and results, focusing on the distributions of properties over different regions of the landscape of string vacua and assessing the phenomenological implications. The topics we discuss include: an overview of how moduli are stabilized and how vacua are counted; the applicability of effective field theory; the uses of and differences between probabilistic and statistical analysis (and the relation to the anthropic principle); the distribution of various parameters on the landscape, including cosmological constant, gauge group rank, and SUSY-breaking scale; "friendly landscapes"; open string moduli; the (in)finiteness of the number of phenomenologically viable vacua; etc. At all points, we attempt to connect this study to the phenomenology of vacua which are experimentally viable.
\end{abstract}

January 2006 


\section{Contents}

1 Introduction $\quad 3$

1.1 Probabilistic vs. statistical analysis . . . . . . . . . . . . . . . . 3

1.2 The major caveat - effective field theorv . . . . . . . . . . . . . . . 5

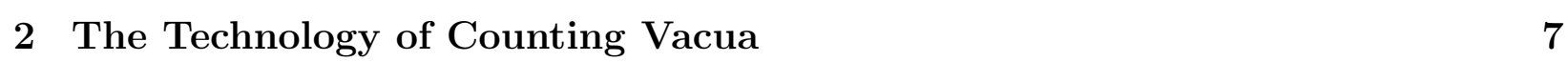

2.1 The Type IIB flux vacua ensemble . . . . . . . . . . . . . . . . . . . 8

2.2 Counting the vacua of the IIB ensemble . . . . . . . . . . . . . . . . 10

2.3 Cosmological constant . . . . . . . . . . . . . . . . . . 13

2.4 Open string moduli $\ldots \ldots \ldots \ldots \ldots$

2.5 Other ensembles . . . . . . . . . . . . . . . . . . . . 16

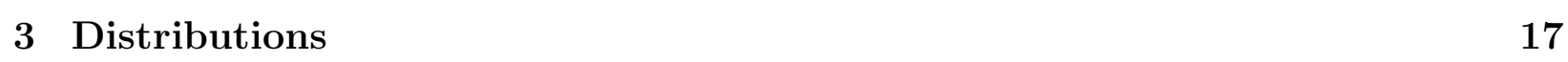

3.1 Gauge group rank . . . . . . . . . . . . . . . . . . . . . . . 17

3.2 Fluxes and moduli . . . . . . . . . . . . . . . . . . . . . . 19

3.3 "Friendly landscapes" and the scanning of parameters . . . . . . . . . . . . 20

3.4 Supersvmmetry breaking scald . . . . . . . . . . . . . . . . . . 22

4 Distributions of Standard Model-like Vacua $\quad 27$

4.1 Standard Model constructions . . . . . . . . . . . . . . . . . . . . . . 28

4.2 Some results . . . . . . . . . . . . . . . . . . . . . . . . . . . . . . 29

4.3 Standard Model phenomenologv considerations . . . . . . . . . . . . 30

5 Discussion - an Infinity of Vacua 


\section{Introduction}

For many years, string theory (or, more generally, M-theory) has been a promising avenue for the study of quantum gravity. In addition, it is a possible theory which could unify the fundamental forces experimentally observed in the real world. One of the most intriguing aspects of string/M-theory is that it is a theory with no free parameters. All low-energy parameters really arise as fields in string theory, whose values are determined dynamically. This raises the interesting prospect that perhaps the dynamics of string theory can uniquely determine the properties of the real world.

Unfortunately, this hope has slowly given way to the belief that there are in fact many different string theory solutions, each of which yields a different low-energy physics. This set of many vacua is often called the "string landscape." Thus, the question of how the parameters of the Standard Model are set is simply replaced by the question of which string vacuum we live in.

In fact, it has seemed clear for quite some time that there are an infinite number of string theory solutions. Examples of infinite classes of vacua which are believed to be stable include Type IIA string theory in flat space, with 32 supersymmetries and the dilaton as a continuously tunable parameter. Other examples include Type IIB string theory in an $A d S_{5} \times S^{5}$ background with arbitrary integral flux, and various $N=2$ compactifications on Calabi-Yau manifolds (where the vacua are parameterized by moduli spaces).

In all of these examples, however, the low-energy physics contains extended, unbroken supersymmetry. As a result, these vacua cannot describe the real world. It may still be hoped that the constraints of supersymmetry breaking force us into a unique vacuum. However, growing evidence suggests that there are in fact many vacua with no moduli and with weakly broken supersymmetry [1, 2, 3, 4, 15, 6, 17, 8, 9, 10, 11, 12, (but see also [13, 14, for evidence of models with supersymmetry breaking at higher energy scales).

Here we attempt to review some of the arguments in favor and against the existence of this landscape of vacua. We also review qualitative and quantitative statistical results as well as their physical implications and phenomenological applications.

\subsection{Probabilistic vs. statistical analysis}

Before getting into the details of how these vacua are constructed, we should first discuss how this information can be used. Broadly, there are two types of questions which one 
can address by studying the landscape. A "what" question asks which vacuum we live in, but a "why" question asks why we live in this vacuum as opposed to any other consistent vacuum. The distinction between these two questions is related to the distinction between probabilistic and statistical analysis of the landscape.

A statistical analysis of the landscape is an attempt to count the number of vacua which exist in controllable classes and estimate the distributions of properties among these vacua. This type of an analysis can be quite useful in relation to the "what" question, because it addresses the point of whether vacua exist whose properties match the real world to within experimental precision. If one can show that it is statistically likely that a vacuum exists whose low-energy properties are consistent with the real world, then it is likely that we actually have a vacuum worth looking for [15]. Whether one can actually find such a vacuum is, of course, a separate challenge which likely requires a much more sophisticated understanding of the mathematics of string compactification than we currently have. But even so, a statistical analysis of controllable models gives one an idea of where to look for viable models, or at least a clue as to whether they likely exist.

However, this statistical analysis makes no comment whatsoever on how nature selects a vacuum. This analysis studies only the statistical distribution of properties among consistent controllable vacua, irrespective of how a vacuum is chosen. The goal of this analysis is to identify the properties of as many vacua as possible which are phenomenologically viable; statistics of this subset are the statistics of a set of vacua, not necessarily of the real world. Indeed, we do not know that nature must choose one of these vacua. It is simply an experimental observation that nature may have chosen one of them, given what we know at the moment.

A probabilistic analysis, however, would seek to determine from this set of vacua the likelihood that any particular set of low-energy physics is observed. The idea is essentially to place a probability measure on the landscape, which determines the likelihood that any particular vacuum is realized. This measure, convolved with the statistical distribution of properties on the landscape, would determine the likelihood that certain low-energy properties are realized by nature.

One example of a type of probabilistic measure is simply to assert that every isolated vacuum is equally likely. Another example would be to convolve this with an anthropic principle, which states that the only vacua which are acceptable are those for which it is possible that life can exist. Thus far, we have made little progress in actually determining a realistic probability measure (though for some interesting results, see [16]). There does not 
appear to be a clear approach to determining a selection principle which is narrow enough to select a unique vacuum. However, the statistical analysis of controllable vacua is quite well-defined and is an area where clear and concrete progress can be made. As such, we focus mostly on statistical analysis.

This is by no means an attempt at a comprehensive review of work on the landscape. Instead, we attempt to survey only one thread of this field, namely the study of distributions of properties among sets of (to varying degrees) phenomenologically viable vacua [22, 23, 14]. In section 2, we review the technology used to count flux vacua in various ensembles. In section 3 , we use this technology to find the distribution of some low-energy parameters among the set of vacua in various controlled constructions. In section 4, we access phenomenologically interesting questions about string constructions of the Standard Model with this technology. We conclude in section 5 with some interesting questions accessible by this type of analysis.

\subsection{The major caveat - effective field theory}

Before proceeding, we should discuss one of the major caveats of this entire analysis ... the reliance on effective field theory. It is quite difficult to construct consistent string backgrounds without supersymmetry, and only in very specific constructions can one find string backgrounds with no moduli. Instead, the study of the landscape has focussed on an effective field theory approach. The basic idea is to look at 10-d string theory compactified on an orientifolded Calabi-Yau 3-fold, yielding a 4d theory with $N=1$ supersymmetry, expanded about a flat background. One can then derive a low-energy effective field theory ( $N=1$ SUGRA) which describes scattering in this flat string background. The crucial jump is to then look for other solutions to this effective action which are not asymptotically equivalent to the string background from which the effective field theory was derived. Thus, it is perhaps more accurate to say that we are not in the business of constructing string vacua, but rather of string-derived effective field theory vacua.

It is an open question whether or not this process is valid [17, 18. There is evidence to suggest that string theories formulated in different backgrounds are described by different Hamiltonians, not by different states of the same Hamiltonian. This might suggest that one cannot begin with a description of string scattering in one background and bootstrap that into a description of strings in another background. One can certainly consider examples where the potential barrier between the original flat supersymmetric string solution and the new effective field theory solution is small relative to the Planck scale. In such a case, one expects effective field theory to be valid, at least over a small enough distance scale. But 
for a large enough bubble of false vacuum, the mass of the domain wall is large enough that it appears to the external observer that a black hole has formed [19]. The external observer attempting to find the inflating region reaches a singularity before reaching an inflating region. This intuition would also seem to suggest that we cannot view large bubbles of an inflating region as excitations of the theory which had the original flat solution.

However, the fact that de Sitter solutions cannot be reached as excitations of Minkowski solutions does not necessarily indicate that they do not live as states in the same theory. Indeed, a similar result was shown in [20], where it was argued that inflating space-times can never result from an initial pure state, because the inflating space-time is necessarily described in the dual gauge theory by a mixed state and cannot arise from a pure-state through unitary evolution ${ }^{2}$. But despite the fact that this mixed state cannot result tunneling or scattering in a pure state, both the mixed states and pure states are clearly states of the same quantum Hamiltonian. Indeed, if there really is a background independent formulation of quantum gravity, then it seems likely that there should be a single quantum theory whose Hilbert space describes all possible string backgrounds. The expansion about two different fixed backgrounds may be contained within two subsectors which are disconnected in some limit, but one would imagine that the full theory should see them both.

And it is certainly the case that, beginning with an effective field theory derived from a string solution, one can in some cases find new string backgrounds with different asymptotics. An example is Type IIB string theory in $A d S_{5} \times S^{5}$. One still does not really know how to quantize Type IIB string theory in the presence of non-trivial RR-fluxes. As such, one cannot demonstrate that $A d S_{5} \times S^{5}$ is a consistent background for the Type IIB string. Instead one considers Type IIB supergravity, which is the low-energy effective field theory derived from the scattering of string in Type IIB string theory in flat space. One then discovers that this low-energy effective action has other classical solutions with supersymmetry, including $A d S_{5} \times S^{5}$. The consistency of Type IIB string theory in the $A d S$ background has since been given much stronger support by the $A d S / C F T$ correspondence, but the original motivation (confirmed by $A d S / C F T$ ) came from effective field theory arguments quite similar to those used in analysis of the landscape.

Of course, in this case the solution has a large amount of supersymmetry. However, there seems to be no obvious reason why the arguments against the effective field theory approach should fail if there is supersymmetry. Indeed, one of the arguments against this effective field theory approach is that, according to $A d S / C F T$, string theory in an $A d S_{5} \times S^{5}$ background

\footnotetext{
${ }^{2}$ Cosmology in the context of AdS/CFT was also discussed in [21].
} 
with quantized flux $N_{1}$ is described by a Hamiltonian which is different from the Hamiltonian which describes $A d S_{5} \times S^{5}$ with flux $N_{2}$ (they are both described by $N=4 D=4 \mathrm{SYM}$, but with different gauge group rank). Nevertheless, they all appear as classical supersymmetric solutions to the equations of motion of the low-energy theory derived from flat-space.

By no means is this question resolved, and one would certainly hope to develop a more rigorous demonstration of consistent non-supersymmetric backgrounds which are phenomenologically viable. However, given the difficulty in achieving this aim and the considerations mentioned above, we content ourselves for the moment with accepting the effective field theory approach at face value.

\section{The Technology of Counting Vacua}

Many different ensembles of vacua have been studied 1, 2, 3, 4, 5, 6, 17, 8, 9, 10, 24, but we focus on IIB flux vacua arising from orientifolded Calabi-Yau compactification, as this is the version on which most work has been done. At a fundamental level, we are searching for vacua which are phenomenologically viable, given current experimental data. As a first step, we search for 4-d vacua with no moduli and broken supersymmetry. Of course, generic supersymmetry-breaking yields potentials for the scalars, lifting all moduli. However, without the control of supersymmetry, generic potentials can cause one or more scalars to run to infinity, destabilizing the solution. In particular, one expects that the vacuum energy of a non-supersymmetric solution contributes a potential term which tends to cause the size moduli to run to infinity, causing decompactification of the solution [25, 26].

Our aim is then to find supersymmetric vacua in which as many moduli as possible are fixed, and then introduce supersymmetry breaking at a scale much lower than the masses of the scalars. Because the scalars are fixed in a supersymmetric vacuum, we have good control over the potential and can be confident that there are no destabilizing corrections. Once we introduce supersymmetry breaking at a lower scale, we can still be confident that the solution is stable, because the masses of the scalars are much larger than the supersymmetry breaking scale. The scalar vevs might shift a little, but they cannot be destabilized.

It may seem a bit strange to refer to "supersymmetric" vacua, when a necessary part of our construction is in fact to break supersymmetry. To be more concrete, we imagine an effective potential which we can write in the form

$$
V=V_{\text {large }}+V_{\text {small }}
$$


where there is a controlled limit in which $V_{\text {small }} \ll V_{\text {large }}$. We can then write

$$
\tilde{V}=V_{\text {large }}+\lambda V_{\text {small }}
$$

Solutions to the equations of motion are now parameterized by $\lambda$. We say that a family of solutions is "almost supersymmetric" if the $F$-term equations ${ }^{3}$ are solved when $\lambda=0$. Once we move to $\lambda \neq 0$, the solution will deform and the $F$-term equations may no longer be satisfied. Nevertheless, the corrections to the solution are small, provided we are in the limit where $V_{\text {small }} \ll V_{\text {large }}$.

\subsection{The Type IIB flux vacua ensemble}

With this idea in mind, the plan is to begin with Type IIB string theory compactified on an orientifolded Calabi-Yau 3-fold. Much of the quantitative analysis of this set-up was pioneered in [8, 1, 30, 31, 32, and we use their notation. The CY compactification preserves 8 real supersymmetries, with the orientifold projection reducing this number to 4 . The lowenergy effective field theory describing this compactification is $N=1 D=4$ supergravity. This approximation is valid in the limit $g_{s} \ll 1, R \gg l_{s}$, where $R$ is a size modulus for the compact dimensions. In the end, we will consider only those solutions where the moduli are fixed in this self-consistent regime. The massless scalar fields are the complex structure moduli, the Kähler moduli and the axio-dilaton.

The potential for this theory is given by

$$
V=e^{K}\left(D_{i} W{\overline{D^{i} W}}^{i}-\frac{3}{M_{p l}^{2}}|W|^{2}\right),
$$

where $K$ is the Kähler potential and $W$ is the superpotential. Here, $D W$ is a covariant derivative with respect to the moduli, given by $D W=\partial W+W \partial K$. By turning on NSNS and RR fluxes, we can generate a tree-level superpotential of the form 33

$$
W_{\text {tree }}=\int G \wedge \Omega
$$

\footnotetext{
${ }^{3}$ One might worry that, even if the $F$-terms vanish, there may be non-trivial $D$-terms which would break supersymmetry, perhaps badly. But by the general arguments of 27, 28, 29, the gauge invariance of $N=1$ $D=4$ supergravity implies that the $D$-terms are of the form

$$
2 \Re f^{a b} D_{b}=\frac{\imath k^{a i} D_{i} W}{W}
$$

where $f$ is a gauge coupling and $k^{a i}$ generates Killing symmetries of the Kähler metric. From this, one sees that the $D$-terms can be non-vanishing only if an $F$-term is non-vanishing as well. So we need only check for solutions to the $F$-term equations. Note that once we break supersymmetry, we may still have $|D| \gg|F|>0$; this corresponds to standard $D$-term breaking in global supersymmetry.
} 
where $G$ is the complexified 3-form flux and $\Omega$ is the holomorphic 3-form. $G$ depends on the axio-dilaton and $\Omega$ depends on the complex structure moduli. These moduli are then fixed by this potential, with masses that scale as $m \sim \frac{l_{s}^{2}}{R^{3}}$. In fact, the $F$-term equations $(D W=0)$ for the complex structure moduli can only be satisfied when the complex structure moduli assume values such that the fluxes are imaginary self-dual (ISD). This means that the holomorphic structure of the fluxes must be of the form $(2,1)$ or $(0,3)$.

This leaves only the Kähler moduli. At tree-level, the no-scale structure of the superpotential implies that the potential cannot fix the Kähler moduli. ${ }^{4}$ But non-perturbative corrections to the superpotential and corrections to the Kähler potential 34] (both perturbative and non-perturbative) break no-scale structure generically. We will find that the non-perturbative corrections to the superpotential will be the dominant correction, and can give masses to the Kähler moduli of order $\sim \frac{1}{l_{s}} e^{-\frac{R}{l_{s}}}$. These corrections can arise from gaugino condensation $^{5}$ or from D3-instantons, for example.

We can demonstrate how this can work. When the complex structure moduli and axiodilaton are fixed at tree-level, the superpotential is fixed at some value $W_{0}$, which we assume to be negative. If we also have $\left|W_{0}\right| \ll M_{p l}^{2}$, then all corrections to the Kähler potential, both perturbative and non-perturbative, are dominated by the non-perturbative corrections to the superpotential. As such, these are the only corrections we consider. The corrected superpotential is of the form

\footnotetext{
${ }^{4}$ It is worth discussing this point in a bit of detail. No-scale structure arises from a theory with a superpotential which is independent of the Kähler moduli, and with a Kähler potential for the Kähler moduli given by $K=-3 \ln [\imath(\rho-\bar{\rho})]$ (in the case of only one volume modulus). In this case, any solution of the equations of motion has $D_{\rho} W \overline{D^{\rho} W}=3|W|^{2}$, and thus positive semi-definite potential $V$. The remaining $F$-term equations are $m$ equations for $m$ unknowns, where $m$ is the number of remaining moduli. If these equations are solved, then $V=0$ and the Kähler moduli are not fixed (regardless of whether or not the $F$-term equations for the Kähler moduli are satisfied). Because the superpotential is independent of the Kähler moduli, we also find that supersymmetric solutions (those which satisfy the $D W=0$ for all moduli) have $W=0$. If there are corrections to the Kähler potential, then $V \geq 0$ will no longer be necessary. But as long as the superpotential is uncorrected, we still find $W=0$ for supersymmetric solutions. If the superpotential receives non-perturbative corrections which depend on the Kähler moduli, however, then we will generically find supersymmetric solutions with $D W=0, W \neq 0, V \neq 0$.

Note that $W=0$ is not compatible with generic ISD fluxes. In particular, non-trivial $(0,3)$ fluxes necessarily force the superpotential to be non-zero. (2,1) ISD fluxes are compatible with the $F$-terms equations and the condition $W=0$. The breaking of no-scale structure implies that $(0,3)$ fluxes can also result in supersymmetric $A d S$ vacua, rather than non-supersymmetric vacua.

${ }^{5}$ There are in fact two different ways in which one may model gaugino condensation in $N=1$ SUGRA. We may either consider it as an explicit vev for an $F$-term, or as a term in the superpotential which is exponential in the volume modulus. The first method is applicable in situations where gaugino condensation breaks SUSY. Of course, in such cases the second method will also reveal that, for appropriate $K$ and $W$, SUSY is broken. More generically [35], though, the first method can be used to show that SUSY is unbroken after gaugino condensation. It is important to keep this distinction in mind, as both methods appear in the literature. In this review, we will represent gaugino condensation via an exponential term in the superpotential.
} 


$$
W=W_{0}+A e^{-a \sigma},
$$

where $\sigma$ is a real Kähler modulus (we assume only one for the moment, and we set the axion to zero) and $A$ and $a$ are constants. The potential arising from this superpotential fixes the Kähler modulus at finite vev. Note however, that consistency of this supergravity approximation requires that $\sigma \gg 1$. If we imagine that the correction arises from a single instanton, then this form is reliable only when $a \sigma>1$. But if $W_{0}<0$ and $\left|W_{0}\right| \ll M_{p l}^{2}$, then these conditions can be satisfied and our solution is self-consistent. ${ }^{6}$

Note that we have not actually shown that the Kähler moduli are fixed 36]. Instead, we have shown that certain types of corrections, which can appear in a wide variety of models, will fix those moduli. In fact, several specific examples have been exhibited in which the Kähler moduli are explicitly shown to be fixed by these types of corrections [11, 12, 4, .

It is at this point that one would examine the types of additional supersymmetry-breaking contributions which can also be included 1, 37, 38, ${ }^{7}$ An example would be to turn on a small amount of IASD flux 38. Another example would be the introduction of anti-D3-branes. As argued in [1, 8, 39], the appearance of fluxes leads to a warping of the compactification, and the potential tends to force the anti-D3-branes to the warped end of the throat, potentially generating a hierarchy. One could also rely on non-perturbative corrections [37] to break supersymmetry.

\subsection{Counting the vacua of the IIB ensemble}

Given that we know the form of the potentials, we can now quantitatively estimate the number of vacua which we can generate. By vacua, we mean a choice of fluxes to turn on

\footnotetext{
${ }^{6}$ In actuality, the expectation values of moduli are really determined by extremizing the full potential as a function of all moduli, not by the procedure described above in which the light fields are truncated while the heavy fields are integrated out, with the light fields then reintroduced. If the complex structure moduli are integrated out from the full potential, the effective potential for the remaining Kähler moduli will in fact be slightly different from the one described in the procedure above 41, 27, 29, 42, due to the backreaction of the heavy fields. But the essential results of a generic hierarchy of scales and fixing at finite values of the complex structure and Kähler moduli are unchanged.

${ }^{7}$ The breaking of supersymmetry introduces a new subtlety regarding tachyons. Solving the $F$-term equations does not necessarily imply that the potential is at a minimum; some of the masses may in fact be tachyonic. If the solution is supersymmetric, however, these tachyons do not constitute an instability. But in realistic scenarios in which supersymmetry is broken and the vacuum energy is positive, one must be sure to consider only solutions for which the bosonic mass matrix is positive definite. It calculated examples this can be true in an $\mathcal{O}(1)$ fraction of vacua, but in limits (such as near a conifold point) it can approach zero 32 .
} 
and a choice of vevs for the complex structure moduli and axio-dilaton such that the $F$-term equations are satisfied.

The essential point is simply to determine what inputs we can vary, and what constraints we are required to satisfy. Our inputs are the choice of complex structure moduli and the choice of integer-valued fluxes. Our constraints are the $F$-term equations and the cancelation of RR-tadpoles. In the simplest cases, the only non-trivial RR-tadpole condition arises from the cancelation of space-filing D3-brane charge, and can be written as

$$
L_{*}=N_{D 3}+\frac{1}{2} \int F_{R R} \wedge H_{N S N S}
$$

where $-L_{*}$ is the D3-brane charge carried by the orientifold, $N_{D 3}$ is the number of D3branes we have added in by hand, and $L=\frac{1}{2} \int F_{R R} \wedge H_{N S N S}$ is the quantized amount of D3-brane charge induced by turning on the RR and NSNS 3-form fluxes. Given our GVW superpotential, the tree-level equations of motion are satisifed only if our fluxes are imaginary self-dual (ISD) [8, which in turn is sufficient to show that $L>0$. Supersymmetry then requires that $N_{D 3}>0$.

For convenience, we absorb the $e^{K}$ factor in front of the potential into the rescaling $D W(z) \rightarrow e^{\frac{K}{2}} D W(z), \Omega(z) \rightarrow e^{\frac{K}{2}} \Omega(z)$. As a result, we drop the overall factor of $e^{K}$ in front of the potential, but instead find that $D W$ and $\Omega$ are no longer holomorphic functions of the moduli. We denote by $n=h_{-}^{2,1}$ the number of complex structure moduli which survive the orientifold projection. The number of real fluxes which we can turn on is then given by $4 n+4$. We can represent the choice of flux in an integral basis by the vector $\vec{N}$. The charge $L$ induced by the flux is a form which is quadratic in $\vec{N}$. For ease of calculation, we will also end up ignoring the quantization of the flux vector $\vec{N}$, allowing us to replace sums over fluxes with integrals. This approximation is justified [32] in the limit where $L_{*}>n$.

Our strategy $^{8}$ for counting vacua is to integrate the unit element over all choices of complex structure moduli and sum over all possible choices of flux, while using a $\delta$-function to impose the $F$-term equations and using a step function to impose tadpole cancelation. The number of flux vacua $\mathcal{N}$ can thus be expressed as

$$
\begin{aligned}
\mathcal{N} & =\sum_{\text {fluxes }} \int d^{2 n+2} z \delta_{z}^{2 n+2}\left(D_{z} W\right) \theta\left(L_{*}-L\right) \\
& =\int \frac{d \alpha}{\alpha} e^{\alpha L_{*}} \int d^{4 n+4} N \int d^{2 n+2} z e^{-\alpha L} \delta_{D W}^{2 n+2}(D W) \operatorname{det}\left|D^{2} W\right|
\end{aligned}
$$

\footnotetext{
${ }^{8}$ This can be related to the attractor mechanism 32,40 .
} 
Since $L$ is quadratic in $N$, we may rescale $N \rightarrow \frac{N}{\sqrt{\alpha}}$ to get

$$
\begin{aligned}
\mathcal{N} & =\int \frac{d \alpha}{\alpha} e^{\alpha L_{*}} \alpha^{-2 n-2} \int d^{4 n+4} N \int d^{2 n+2} z e^{-L} \delta_{D W}^{2 n+2}(D W) \operatorname{det}\left|D^{2} W\right| \\
& =\frac{\left(L_{*}\right)^{2 n+2}}{(2 n+2) !} \int d^{4 n+4} N \int d^{2 n+2} z e^{-L} \delta_{D W}^{2 n+2}(D W) \operatorname{det}\left|D^{2} W\right| .
\end{aligned}
$$

At this point, we must find a nicer basis for the fluxes. Since the fluxes appear in the superpotential, we can write a basis for the fluxes in terms of the superpotential and derivatives thereof (with respect to the complex structure moduli). This is particularly useful for integrating over the $\delta$-function in a simple manner.

We choose a basis for the fluxes where the coefficients are given by

$$
\begin{aligned}
X & =W \\
Y_{A} & =D_{A} W \\
Z_{I} & =D_{0} D_{I} W
\end{aligned}
$$

where $A=1 \ldots n, I=0 \ldots n$. $A$ is an index which runs over the complex structure moduli, while I runs over those moduli plus the axio-dilaton ('0') as well. In terms of these coefficients, we may write the D3-brane charge induces by the fluxes as

$$
L=|X|^{2}-|Y|^{2}+|Z|^{2} .
$$

We get the expression

$$
\mathcal{N}=\frac{\left(L_{*}\right)^{2 n+2}}{(2 n+2) !} \int d^{2 n+2} z \int d X d^{2 n+2} Z e^{-\left|X^{2}\right|-\left|Z^{2}\right|} \operatorname{det}\left|D^{2} W\right|_{Y=0} .
$$

The det $\left|D^{2} W\right|$ factor arises from the change in normalization of the $\delta$-function which allows it to fix a flux instead of a modulus. This determinant depends on the fluxes $X$ and $Z$, the complex structure moduli and axio-dilaton, and on the geometric data $\mathcal{F}$ of the CalabiYau. Note the way in which the number of vacua factorizes. The prefactor depends on only on very simple topological data $\left(n\right.$ is the number of complex structure moduli and $L_{*}$ is the background charge of the orientifold, which is the same as the Euler character of the Calabi-Yau four-fold which is the $F$-theoretic dual of this compactification). This prefactor essentially counts the number of choices of the flux which are compatible with the RR-tadpole conditions at any given point in moduli space ${ }^{9}$. The integral essentially integrates over a density of vacua in complex structure moduli space.

\footnotetext{
${ }^{9}$ Thus far, we have basically ignored the discreteness of the flux. If $n \gg L_{*}$, many integral flux coefficients vanish and the discreteness cannot be ignored. In this case, one instead expects $\mathcal{N} \sim e^{\sqrt{2 \pi c n L_{*}}}$, where $c$ is a constant 31].
} 
An index density can be defined by removing the absolute value symbols from the determinant, and counts the vacua weighted by a sign. This index density provides a minimum estimate for the number vacua, and one of the reasons for its usefulness is the simple estimate demonstrated in 31,32 :

$$
\rho_{\text {ind }}=\pi^{-(n+1)} \operatorname{det}(R+1 \omega)
$$

where $R$ and $\omega$ are the curvature 2-form and Kähler 2-form respectively on the complex structure moduli space $\mathcal{M}_{C}$. This indicates that string vacua tend to be concentrated in regions of the moduli space of high curvature, such as near a conifold singularity. The correlation between the density of vacua and the curvature of the moduli space is key to developing a correlation between low-energy parameters and the density of vacua. ${ }^{10}$

We have not yet included the non-perturbative corrections to the superpotential. These generate additional $F$-term equations which fix the remaining Kähler moduli. The form of this equation depends on the non-perturbative corrections, but does not seem to depend on the parameters relevant for our original counting (i.e., $n, L_{*}, \mathcal{F}$ ). Indeed, it seems to depend on the tree-level superpotential only through $W_{0}$. As such, this problem essentially factorizes into a product of solutions to the complex structure moduli (and axio-dilaton) Fterm equations and Kähler moduli F-term equations. This counting of solutions to the Kähler moduli equations of motion depends on the exact form of the non-perturbative corrections, so we do not address it in detail. But we shall see that we can gain much statistical knowledge simply from the distribution of solutions of the first set of $F$-term equations.

Finally, remember that we still have some self-consistency requirements, namely that the moduli be fixed in such a way that $g \ll 1$ and $R \gg 1$ (equivalently, $\sigma \gg 1$ ). This analysis is only valid for the fraction of vacua where the moduli happen to be fixed in this self-consistent region. But the distribution of vacua is basically uniform in this region, so restricting to this region does not dramatically reduce the number of vacua 31 .

\subsection{Cosmological constant}

We may similarly study the distribution of the tree-level cosmological constant by counting the flux vacua for which cosmological constant is within a specified range. For a supersymmetric solution where the $F$-term vanishes, the cosmological constant is simply given by

\footnotetext{
${ }^{10}$ There are topological arguments to suggest that this type of index density estimate should be valid beyond the limited scope of Calabi-Yau compactification 31.
} 


$$
\Lambda=-3 e^{K} \frac{|W|^{2}}{M_{p l}^{2}}
$$

What really are seeking is the distribution of $e^{K}|W|^{2}$, which can be written very simply in terms of our fluxes. Indeed, this term is precisely the flux coefficient $X$ which we defined earlier.

We can now count the number of flux vacua whose tree-level cosmological constant is given by $|\Lambda| \leq \Lambda_{0}$. We simply follow the same counting procedure as before, except we include an integration over a parameter $\Lambda$, and a $\delta$-function in our integral to pick out vacua with cosmological constant equal to $\Lambda$. This yields

$$
\mathcal{N}=\int \frac{d \alpha}{\alpha} e^{\alpha L_{*}} \int_{0}^{\Lambda_{0}} d \Lambda \int d^{4 n+4} N \int d^{2 n+2} z e^{-\alpha L} \delta_{D W}^{2 n+2}(D W) \delta\left(\Lambda-|W|^{2}\right) \operatorname{det}\left|D^{2} W\right| .
$$

We can again rescale $N \rightarrow \frac{N}{\sqrt{\alpha}}$, remembering now to rescale $\Lambda$ and $\Lambda_{0}$ by $\frac{1}{\alpha}$ as well. We then find

$\mathcal{N}=\int \frac{d \alpha}{\alpha} e^{\alpha L_{*}} \alpha^{-2 n-2} \int_{0}^{\Lambda_{0} \alpha} d \Lambda \int d^{4 n+4} N \int d^{2 n+2} z e^{-L} \delta_{D W}^{2 n+2}(D W) \delta\left(\Lambda-|W|^{2}\right) \operatorname{det}\left|D^{2} W\right|$.

The vacuum density on moduli space is now a function of the parameter $\Lambda_{0}$ as well. If $\Lambda_{0}$ is small enough, then the vacuum density is approximately constant with respect to $\Lambda$ in the range $0 \leq|\Lambda| \leq\left|\Lambda_{0}\right|$ and is equal to it its value at $\Lambda=0$. We get

$$
\begin{aligned}
\mathcal{N} & =\int \frac{d \alpha}{\alpha} e^{\alpha L_{*}} \alpha^{-2 n-1} \Lambda_{0} \int d^{4 n+4} N \int d^{2 n+2} z e^{-L} \delta_{D W}^{2 n+2}(D W) \delta\left(|W|^{2}\right) \operatorname{det}\left|D^{2} W\right| \\
& =\Lambda_{0} \frac{\left(L_{*}\right)^{2 n+1}}{(2 n+1) !} \int d^{4 n+4} N \int d^{2 n+2} z e^{-L} \delta_{D W}^{2 n+2}(D W) \delta\left(|W|^{2}\right) \operatorname{det}\left|D^{2} W\right| .
\end{aligned}
$$

Making the change of flux basis as before, and integrating over the flux coefficient $X$ as well gives us

$$
\mathcal{N}=\Lambda_{0} \frac{\left(L_{*}\right)^{2 n+1}}{(2 n+1) !} \int d^{2 n+2} Z \int d^{2 n+2} z e^{-|Z|^{2}} \delta_{D W}^{2 n+2}(D W) \operatorname{det}\left|D^{2} W\right|_{X=Y=0}
$$

This expression for the number of flux vacua is similar to the expression we found earlier, but with two notable differences. The vacuum density on complex structure moduli space is slightly different. But more importantly, the previous scaling of $L_{*}^{2 n+2}$ is now replaced by a scaling of $L_{*}^{2 n+1} \Lambda_{0}$. In particular, the number of flux vacua with tree-level cosmological constant between $\Lambda_{0}$ and 0 scales linearly with $\Lambda_{0}$, when $\Lambda_{0}$ is small. 


\subsection{Open string moduli}

So far, we have only discussed the fixing of closed string moduli. If there are space-filling branes in the theory, then the low energy effective field theory also has scalar fields arising from open strings. These scalars are in some ways less problematic than closed string scalars. It is important that all of these scalars get a mass for phenomenological reasons, and supersymmetry breaking will generically allow potentials which can give mass to all of these scalars. We wanted to make sure that the closed string scalars got mass at an even higher scale in order to ensure that the generic potentials do not ruin the compactification. The endpoint of open string scalar decay is usually much more controllable, however, and can be understood in terms of brane decay. In any case, we do not need to worry about the runaway problems which plague closed string moduli, since the open string scalars are compact. As a result, we can rely on supersymmetry breaking effects, if needed, to generate open string masses. Indeed, these effects are needed to fix moduli in realistic cases.

We can broadly divided up open string scalars into two groups: those which have vectorlike gauge transformations, and those which do not. If a chiral multiplet has vector-like transformations, then the entire multiplet can pair up with another multiplet and gain mass. But if the multiplet's gauge transformations under the low-energy gauge group are not vector-like, then the fermionic component cannot get mass. This implies that its scalar superpartner also cannot get mass unless supersymmetry is broken ${ }^{11}$. Since the Standard Model has fermions which are chiral under the Standard Model gauge group, the associated squarks and sleptons can only get masses set at the supersymmetry breaking scale.

More generally, non-trivial dependence of the superpotential on the open string scalars may help avoid certain difficulties in realizing inflation on the landscape 43 . It was shown in 43 that models of inflation where the inflaton potential arose from brane-antibrane interactions in a warped geometry could satisfy the slow-roll conditions. However, this condition would be spoiled by the simple mechanism for fixing the Kähler moduli described above (a non-perturbative correction to the superpotential which introduces dependence on the Kähler modulus $\rho$ ). This problem may be alleviated, however, by a fine-tuned dependence of the superpotential on the open-string scalar which acts as the inflaton.

There are a variety of effects which can give rise to masses for open string scalars, both SUSY-breaking and SUSY-preserving. For example, ISD fluxes can give SUSY-preserving

\footnotetext{
${ }^{11}$ In fact, the scalars may have an effective mass at the cosmological constant scale, even if supersymmetry is unbroken. As this scale is already low enough to be ruled out by experiment, the larger supersymmetry breaking scale still controls the mass of these scalars in realistic models.
} 
masses to vector-like chiral multiplets living on D7-branes (though not D3-branes). IASD fluxes give soft supersymmetry-breaking masses to some open string scalars. ${ }^{12}$ Also, the open string scalars will appear in the D-term potential along with FI-terms, which depend on the closed string Kähler moduli. But a generic type of supersymmetry breaking added to any supersymmetric compactification will also generate a generic potential for these scalars whose scale is set by the SUSY-breaking scale.

\subsection{Other ensembles}

One may in a similar way construct flux vacua in other string settings. Each of these settings provides a different statistical distributions. Recently, significant work has been done on the study of flux vacua of Type IIA compactified on an orientifolded Calabi-Yau three-fold [24, 3, 4] ${ }^{13}$. It appears to be much easier to find explicitly controlled solutions in these examples, but with statistical distributions which are much less broad. We will not describe these constructions or the relevant statistical analysis in detail; we simply note the important results and refer the reader to the original sources for more information.

In the ensemble of Type IIA flux vacua on orientifold CY's, we are at liberty to turn on the RR-form fluxes $F_{0}, F_{2}$ and $F_{4}$ as well as the NSNS-form $H_{3}$. The potential which fixes the complex structure moduli depends only on the various RR-fluxes $\left(F_{0,2,4}\right)$ while the Kähler moduli are fixed by the NSNS-flux $H_{3}$. The choice of $F_{0}$ and $H_{3}$ is fixed by the constraints of tadpole cancelation, but the choice of $F_{4}$ is arbitrary. This choice gives us an infinite number of flux vacua. However, as $\left|F_{4}\right| \rightarrow \infty$, these vacua will concentrate at the limit of small coupling and large volume. As a result, there are an infinite number of controllable vacua, but not phenomenologically viable vacua (for large enough volume of the compact space, these vacua can be ruled out by experiment).

In this construction, one finds that the number of vacua in which the size of the compact manifold is $\leq R_{*}$ scales as $\mathcal{N}\left(R \leq R_{*}\right) \sim\left(R_{*}\right)^{4}$. Similarly, the number of vacua with small cosmological constant scales as $\mathcal{N}\left(|\Lambda| \leq\left|\Lambda_{*}\right|\right) \sim\left(\left|\Lambda_{*}\right|\right)^{-\frac{2}{9}}$. The vacua of this ensemble are

\footnotetext{
${ }^{12}(0,3)$ ISD fluxes would also give soft SUSY-breaking masses to open string scalars if no-scale structure were unbroken. But as we reviewed earlier, one expects that generic corrections to the superpotential and Kähler potential will break no-scale structure.

${ }^{13}$ Although this ensemble might appear to be simply the mirror of our IIB ensemble, it is in fact somewhat different. The difference arises from the fluxes of IIB and IIA, which do not map into each other in a simple way under mirror symmetry. Instead, the fluxes map into geometric fluxes, and may result in mirrors which are non-Kähler, and perhaps even non-geometric [5, 44]. We will not discuss this further, except to note that when all such additional compactifications are included in the ensemble, then we expect the distributions of properties in the extended IIA ensemble to be identical to that of the mirror extended IIB ensemble.
} 
most highly populated at small coupling, small cosmological constant and large compact volume.

Another ensemble subject to recent interest is the set of flux vacua arising from a compactification of $M$-theory on a $G_{2}$ manifold with 4 -form fluxes turned on [2, 45].

\section{Distributions}

Having seen how to develop the technology for counting flux vacua in a variety of string compactifications, we would like to use this technology to study the distribution of lowenergy observables over the set of vacua. The main points we need to understand are how low-energy parameters depend on the fluxes and moduli, and how the density of vacua correlate with the choices of flux and moduli.

Note that this is not a survey of the distribution of low-energy parameters over all vacua. It is only a survey of the distribution over certain controllable sets of vacua. These distributions may be representative of the distribution over all vacua, but there is not yet sufficient evidence to suggest that this possibility is correct or incorrect. We can only say so far that we are studying the distribution of parameters in a set of vacua which model-builders can potentially construct.

\subsection{Gauge group rank}

As an example, one can compute the distribution of gauge group rank arising from D3-branes in the IIB ensemble using the basic counting methodology we have already reviewed [46]. The basic point is that the number of flux vacua which we have found is given by

$$
\mathcal{N}=c_{C Y} L_{*}^{2 n+2}
$$

where $c_{C Y}$ is a constant which depends on the structure of the Calabi-Yau, $L_{*}$ is the D3-brane charge of the orientifold plane and $n$ is the number of complex structure moduli. From our previous derivation of this result, one sees that the $L_{*}$ dependence arises entirely from the step function which was inserted to enforce the condition that the charge arising from the fluxes be bounded by $L_{*}$. Thus, by an appropriate choice of the step function, we find that the number of flux vacua with flux charge less than any integer $L$ is given by $\mathcal{N}=c_{C Y} L^{2 n+2}$. Since the number of D3-branes which must be added by hand (to cancel the RR tadpoles) is simply given by $N_{D 3}=L_{*}-L$, we can easily compute the average number of D3-branes in this ensemble of vacua: 


$$
\begin{aligned}
\left\langle N_{D 3}\right\rangle & =L_{*}-\frac{1}{c_{C Y} L_{*}^{2 n+2}} \int_{0}^{L_{*}} d L L \partial_{L}\left(c_{C Y} L^{2 n+2}\right) \\
& =\frac{L_{*}}{2 n+3} .
\end{aligned}
$$

Here we see that the ensemble average is entirely independent of the details of the CalabiYau, including its singularity structure, geometric data, etc. Instead, it depends entirely on topological properties of the compactification.

Using this average, one may characterize the entire distribution. In particular, the fractional density of vacua as a function of the rank $R$ of the gauge group is given by

$$
\begin{aligned}
\rho & =-\partial_{R}\left(\frac{L_{*}-R}{L_{*}}\right)^{2 n+2} \\
& \sim-\partial_{R}\left(e^{-\frac{R}{\left\langle N_{D 3}\right\rangle}}\right) \\
& \sim \frac{1}{\left\langle N_{D 3}\right\rangle} e^{-\frac{R}{\left\langle N_{D 3}\right\rangle}} .
\end{aligned}
$$

One can make a similar calculation in the limit where the tree-level cosmological constant is small, and one finds that the fractional rank distribution has the same form of exponential dropoff, but instead characterized by a modified average

$$
\left\langle N_{D 3}\right\rangle_{\text {smallc.c. }}=\frac{L_{*}}{2 n+2} .
$$

From this we see that there is indeed a small correlation between the rank of the gauge group (arising from D3-branes) and the tree-level cosmological constant. This correlation goes as $\frac{1}{n}$, and thus becomes small in the limit where the number of complex structure moduli is much larger than the $L_{*}$, the background D3-charge of the orientifold planes. This type of correlation between different low-energy observables on the landscape (in this case, between cosmological constant and gauge group) is very interesting from a phenomenological point of view. Strong correlations, combined with experimental input, can provide a very useful guide for string model-building.

Note also that we have only considered the fixing of closed string moduli. To truly count the number of vacua, we should convolve this distribution with the density of solutions to the equations of motion for the open string moduli as well. Douglas 30 has argued that the number of solutions to the equations of motion for open string moduli should go as $c^{R}$, where $c$ is a constant and $R$ is the rank of the open string gauge theory. A more detailed study of the distribution of vevs for open string moduli is presented in [47. 
The choice of which distribution one uses depends on the type of question one asks. For example, suppose a model-builder was attempting to decide if a particular choice of CalabiYau manifold would be a good choice for study, based on the likelihood that it contains a model with suitable hidden sector gauge group at high energy. In this case, one would first worry about the the closed string dynamics which leads to a choice of gauge group, and only later would concern oneself with the distribution of open string vacua. On the other hand, if one is studying the total number of vacua, it would make sense to study the entire distribution of solutions for open and closed string scalars.

\subsection{Fluxes and moduli}

More generally, we would like to understand how our choice of integral flux couples to our possible choice of moduli through the $F$-term equations. This determines how broadly lowenergy parameters are distributed. A useful parameter for estimating our ability to fix moduli is 4 ]

$$
\eta=\frac{\# \text { of real participating fluxes }}{\# \text { of real moduli }}
$$

where the "participating" fluxes are those which enter the terms of the potential which involve the set of moduli in question. This ratio is useful because we may think of the $F$-term equations for $q$ moduli as really being $q$ equations for the participating fluxes, with the moduli being parameters. The more participating fluxes there are (i.e., higher $\eta$ ), the easier it is to solve the $F$-term equations for arbitrary values of the moduli. The smaller the number of participating fluxes (smaller $\eta$ ), the more we must fine-tune the moduli to allow $F$-term equation solutions, if they can be found at all. Thus, for $\eta>1$ we expect to be able to fix the moduli with a broad distribution of possible solutions by an appropriate choice of flux. For $\eta \sim 1$, we still expect that we can probably fix almost all moduli, but the discreteness of our choice of flux might limit the range of moduli values we can achieve by tuning fluxes. For $\eta<1$, however, we expect that we only have enough freedom to fix a fraction of the moduli.

Our original IIB ensemble yields $\eta_{I I B, c p x} \sim 2$, when we look only at the complex structure moduli. This indicates that we can easily fix the complex structure moduli over a large range of the complex structure moduli space. However, the fluxes do not let us fix the Kähler moduli at all; other non-perturbative effects are required for this.

In the Type IIA case, we find that for the complex structure moduli and Kähler moduli 
we have $\eta_{I I A, c p x} \sim \frac{1}{2}$ and $\eta_{I I A, k} \sim 1$ respectively. This tells us that we can fix all the Kähler moduli, but not along a particularly broad distribution. Only half of the complex structure moduli are fixed, however. The remaining half are unfixed axions. For each such axion, there is a Euclidean D2-instanton which can give mass to the axion. In any case, since the axions are compact scalars, we again will not need to worry about the standard runaway problems of closed string moduli and can thus also rely on supersymmetry-breaking corrections to generate masses for the axions. Note that experimental constraints on the masses of possible axions are looser than those on non-axionic scalars.

In the ensemble of flux vacua obtained by studying $M$-theory compactified on a $G_{2}$ manifold 45], we similarly find $\eta_{M} \sim \frac{1}{2}$, again implying that we can only fix half of the moduli.

\section{3 "Friendly landscapes" and the scanning of parameters}

We have found that in the IIB ensemble, certain observables such as $W$ (which is related to the cosmological constant and the gravitino mass) have very broad distributions on the landscape. This is due to the high value of $\eta$ in this ensemble; there are enough fluxes so that for a broad distribution of possible values of $W$, one can find a choice of fluxes and moduli which satisfy the $F$-term equations.

But this is based on a hidden assumption, namely that the GVW superpotential generates somewhat generic couplings for the moduli. If all the moduli couple to each other in the potential, then we can expect our intuition to hold. This manifests itself in our formalism as a condition on the matrix det $\left|D^{2} W\right|$, which depends on our flux coefficients $X, Y_{A}$ and $Z_{I}$ (in this basis, for example, $X=W$ ), the complex structure moduli and on the geometric data of the Calabi-Yau manifold. The geometric data encode for us the way the moduli couple to each other through the GVW superpotential. Given that the periods of a Calabi-Yau are generically quite complicated functions of the potential, one generically gets a potential in which the complex structure moduli couple to each other, and the extrema of $W$ are broadly distributed. This corresponds to a relatively unpeaked dependence of $\operatorname{det}\left|D^{2} W\right|$ on $X$.

However, one could study models where this matrix has a highly peaked dependence, and these would provide distributions which defy some of our IIB flux vacua intuition. In some highly decoupled models, for example, one can find masses and couplings which instead have very narrow distributions highly peaked at the ensemble average. The authors of [23] proposed a set of conditions in an effective field theory example which would give such narrow distributions. 
This model is based on the assumption the low-energy scalars of the theory are basically decoupled from each other. For example, the potential of this theory might be written as

$$
V(\phi)=\sum_{i}^{N} V_{i}\left(\phi_{i}\right) .
$$

For simplicity, we assume that all potentials $V_{i}$ are of the same form and are minimized at only two critical points $\phi_{i}^{ \pm}$, such that $V_{i}\left(\phi_{i}^{ \pm}\right)=V_{i}^{ \pm}=\bar{V}_{i} \pm \Delta V_{i}$. Defining $\bar{V}=N \bar{V}_{i}$, we see that distribution of $V$ is given by the standard Gaussian form

$$
\rho(V)=\frac{2^{N}}{\sqrt{2 \pi N} \Delta V_{i}} e^{-\frac{(V-\bar{V})^{2}}{2 \Delta V_{i}^{2}}} .
$$

Indeed, the general form of this Gaussian distribution did not depend on our assumption that all $V_{i}$ were of the same form, or that each had only two minima. The standard arguments of the Central Limit Theorem tell us that if $N$ is sufficiently large, then for any choice of the $V_{i}$ 's the distribution of $V$ will be a Gaussian centered at the average value and with a width determined by the root-mean-square of the spread between the minima of each $V_{i}$. The only requirement for this Gaussian distribution is that the individual potentials $V_{i}$ be independent. In such a case, for fixed values of the complex structure moduli $z_{i}$, one finds that det $\left|D^{2} W\right|$ is highly peaked as a function of $X$.

The point is that, even though this toy model has $2^{N}$ vacua, the value of the potential $V$ is not broadly distributed among them. Indeed, the distribution is a Gaussian which is very sharply peaked at $\bar{V}$.

In a similar way, one can find a broad array of physical observables whose distribution among vacua is sharply peaked, provided they depend on moduli which decouple from each other. For example, if the Higgs field has couplings to the closed string scalars of the form

$$
\sum_{i}^{N} F\left(\phi_{i}\right) h^{\dagger} h
$$

then one would also expect the Higgs mass to have a distribution which is a sharply peaked Gaussian.

In this type of ensemble, the distribution appears to be flat only in a region very close to the average, and the size of this region $\frac{\delta X}{X_{a v g}}$ is only significant if the parameter $X$ in question has an average value which is zero, or very small. There is usually no natural reason for any parameter to have an average value of zero unless there exists a symmetry (perhaps very slightly broken). Thus we would find that certain parameters which were protected by symmetries would appear to "scan" a broad distribution in the flux vacua, while other parameters would exhibit a distribution sharply peaked at a non-zero ensemble average value. 
It may appear that this type of an ensemble, with closed string scalar fields which are very weakly coupled, would rarely occur as a low-energy description of a controllable string theory flux compactification. However, it has been argued that this type of ensemble may not be so rare after all. In [48, the authors impose on the low-energy effective action the constraint that the Planck scale and Kähler metric be radiatively stable, as well as the constraint that the superpotential is well-described by perturbation theory. Assuming these constraints, they find a minimum width for the distribution of $|W|^{2}$ which is consistent with a sharply peaked distribution. Of course, this is only a minimum width for the distribution; the actual width could be much broader. But the fact that the minimum is quite small and there is no clear way of arguing for a broader width suggests that there may exist a sizable class of string flux compactifications for which some low-energy parameters are not broadly distributed among flux vacua.

The authors of [23] raise this possibility as a concrete way of understanding the Weinberg argument 49] for solving the cosmological constant problem via the Anthropic Principle. The point would be that such narrow distributions might explain why one can hold parameters of the Standard Model fixed while only varying the cosmological constant in Weinberg's argument. Given our emphasis on statistics as opposed to selection, we do not focus on this point but rather on fact that there is a unique phenomenology associated with this class of flux vacua, whose statistical distributions are different from what one typically expects of the IIB ensemble.

\subsection{Supersymmetry breaking scale}

One of the questions which we would like to study is the distribution of supersymmetry breaking scales on the landscape (this has already been discussed by several authors [50, 52]). It is important to remember that the question is not necessarily which scale of supersymmetry does string theory "predict." This requires a knowledge of the probability measure on the set of flux vacua, which we do not yet understand. Instead, the questions are: what is the distribution of the supersymmetry breaking scale in different controlled ensembles of vacua, how does it correlate with other observable properties, and how many viable string models do we believe exist with various scales of supersymmetry breaking.

The mathematical technology does not yet exist which permits a definitive answer to the question of how the SUSY-breaking scale is distributed in the complete ensemble of phenomenologically viable vacua. But we can discuss how the supersymmetry breaking scale is distributed in various controlled subensembles. 
As pointed out by Douglas and Susskind, the ensemble of flux vacua with tree-level supersymmetry breaking is likely to be dominated by vacua with high-scale supersymmetry breaking. Note that this ensemble is quite different from the original ensemble which we discussed. In that ensemble, we looked at Type IIB vacua where our solutions solved $D W=$ 0 , where the superpotential $W$ included both the tree-level GVW term and certain nonperturbative corrections. It was then assumed that supersymmetry could be broken later by the addition of small SUSY-breaking terms. Instead, we now consider the case where $D W \neq 0$ even at the tree-level approximation. This ensemble is often referred to as the "nonsupersymmetric" ensemble, even though our previous "almost supersymmetric" ensemble also involved broken supersymmetry at the end of the day. But remembering our earlier criterion, the "almost supersymmetric" ensemble involved solutions for which there was a limiting procedure in which the supersymmetry of the deformed solution was restored. In the non-supersymmetric ensemble, this is not the case.

It is relatively easy to see why the non-supersymmetric ensemble is dominated by highscale supersymmetry breaking. In general there can be many different $F$ and $D$ terms which contribute to supersymmetry breaking, and we find the general formula

$$
M_{\text {susy }}^{4} \sim \sum_{i}^{N_{F}}\left|F_{i}\right|^{2}+\sum_{j}^{N_{D}} D_{j}^{2} .
$$

One might imagine writing the number of non-supersymmetric vacua with supersymmetry breaking scale $M_{\text {susy }}$ as $[50]$

$$
\begin{aligned}
d \mathcal{N}_{M_{\text {susy }}} & =d M_{\text {susy }}^{4} \int d^{2 N_{F}} F \int d^{N_{D}} D \delta\left(M_{\text {susy }}^{4}-\sum_{i}^{N_{F}}\left|F_{i}\right|^{2}-\sum_{j}^{N_{D}} D_{j}^{2}\right) \rho(F, D) \\
& \sim\left(M_{\text {susy }}\right)^{2 N_{F}+N_{D}-1} d M_{\text {susy }}^{2}
\end{aligned}
$$

by assuming at the end that the vacuum density $\rho$ is relatively constant. Such an answer would indicate that the number of vacua can with supersymmetry breaking at the scale $M_{\text {susy }}$ can grow as a high power of $M_{\text {susy }}$. Unfortunately this derivation, though intuitively appealing, is not quite correct[51]. The basic problem is that $\rho(F, D)$ is not relatively constant.

To understand this difficulty, we must remember that there are several further constraints which we must impose to ensure that we are counting valid solutions. In particular, we must demand $V^{\prime}=0$, metastability (the absence of tachyons in the expansion about the extremum), and $\Lambda \ll|F|^{2} \ll M_{p l}^{4}$ (i.e., weak supersymmetry breaking and very small cosmological constant). 
The constraint $V^{\prime}=0$ implies that all of the $F$-terms cannot be set independently. In fact, the $F$-terms generically fill out only a one-complex dimensional space, as opposed to the naive $n_{F}$-complex dimensional space. Essentially, the constraint that the goldstino (which is eaten by the gravitino) have a definite mass requires that $\left\langle F_{i}\right\rangle$ (which must be in the same multiplet) have only one complex degree of freedom. If we write the superpotential as a function of this multiplet $\Phi$ (without loss of generality, we may choose this multiplet so that the minimum of the potential occurs when the scalar component vanishes), we find

$$
W=W_{0}+\alpha \Phi+\frac{\beta}{M_{p l}} \Phi^{2}+\frac{\gamma}{M_{p l}^{2}} \Phi^{3}+\ldots
$$

Our above constraints then imply[51] $\alpha, \beta, \gamma \sim|F| \sim M_{\text {susy }}^{2}$ (we ignore $D$-term contributions here for simplicity). The constraint of very small cosmological constant further implies that $W_{0} \sim|F| \sim \alpha, \beta, \gamma$. We have already seen that $W_{0}$ appears to have a uniform distribution in many examples (in the limit of small $W_{0}$ ) 32. Having imposed the necessary constraints, it thus appears that the parameters $\alpha, \beta$, and $\gamma$ also have distributions which are relatively uniform. We can now write an estimate for the number of vacua with small cosmological constant as

$$
\begin{aligned}
d \mathcal{N}_{M_{\text {susy }}}\left(\Lambda \leq \Lambda_{0}\right) & \sim \frac{d M_{\text {susy }}^{2}}{M_{\text {susy }}^{2}} \int d^{2} \alpha d^{2} \beta d^{2} \gamma d^{2} W_{0} \theta\left(\Lambda_{0}-V\right) \theta\left(\{|\alpha|,|\beta|,|\gamma|\}-M_{\text {susy }}^{2}\right)^{3} \\
& \sim \Lambda_{0} M_{\text {susy }}^{10} d M_{\text {susy }}^{2}
\end{aligned}
$$

One might expect that the fine-tuning necessary to obtain the correct Higgs mass is $\sim \frac{M_{\text {higgs }}^{2}}{M_{\text {susy }}^{2}}$. We thus find that the number of non-supersymmetric vacua with Higgs mass $M_{\text {Higgs }}$ and cosmological constant $\Lambda_{0}$ generically scales with $M_{\text {susy }}$ as $M_{\text {susy }}^{10}$.

The story is different, however, in the "almost supersymmetric" ensemble. The reason is essentially because the $F$-terms in this ensemble are naturally small. If we imagine them arising from gaugino condensation (for example), they would damp exponentially as $M_{\text {susy }}^{2}=$ $M_{p l}^{2} e^{-\frac{c o n s t}{g^{2}}}$. The cosmological constant is given by

$$
\Lambda=M_{\text {susy }}^{4}-\frac{3}{M_{p l}^{2}}\left|W_{0}\right|^{2}
$$

The fraction of states with cosmological constant $\Lambda<\Lambda_{0}$ is then given by the expression [52]

$$
\begin{aligned}
F_{1}\left(\Lambda<\Lambda_{0}\right) & =\int_{0}^{W_{\max }} d^{2} W_{0} P_{W}\left(W_{0}\right) \int_{\ln \left(3\left|W_{0}\right|^{2}\right)}^{\ln \left(3\left|W_{0}\right|^{2}+\Lambda\right)} d\left(g^{2}\right) \frac{1}{g^{4}} P_{g^{2}}\left(g^{2}\right) \\
& \sim \int_{0}^{W_{\max }} d^{2} W_{0} \frac{\Lambda_{0}}{\left|W_{0}\right|^{2}} P_{W}\left(W_{0}\right) \frac{1}{\ln W_{0}^{2}} P_{g^{2}}\left(-\frac{1}{\ln \left(W_{0}\right)}\right)
\end{aligned}
$$


where $P_{W}$ and $P_{g^{2}}$ are the probability densities for $W$ and $g^{2}$ respectively. If these distributions are roughly flat, then in the limit of small $\Lambda$ we find

$$
F_{1}\left(\Lambda<\Lambda_{0}\right) \propto \Lambda_{0} \ln \left(M_{\text {susy }}^{2}\right)
$$

In this ensemble, the growth of the number of vacua as a function of the SUSY-breaking scale (at small $\Lambda$ ) is already less steep than the power-law expansion of the non-supersymmetric ensemble. But we have not yet included any constraints on the Higgs mass. If one restricts to the set of vacua with Higgs mass in the expected range (as well as small $\Lambda$ ), the fraction of vacua with SUSY-breaking scale larger than the intermediate scale is very small. The reason is essentially the same as the standard naturalness argument. If supersymmetry is broken at the scale $M_{\text {susy }}$ and is transmitted to the visible sector by gravitational effects, then the natural scale for the Higgs mass is

$$
M_{n a t}=\frac{M_{s u s y}^{2}}{M_{p l}} .
$$

If the Higgs mass is actually measured at $M_{\text {higgs }}$, then the expected fraction of tuning required is

$$
\eta=\frac{M_{\text {higgs }}^{2}}{M_{\text {nat }}^{2}}=\frac{M_{\text {higgs }}^{2} M_{p l}^{2}}{M_{\text {susy }}^{4}} .
$$

There is no fine-tuning suppression for $M_{\text {susy }} \leq M_{\text {int }}=\sqrt{M_{\text {higgs }} M_{p l}}$. However, for much larger $M_{\text {susy }}$, the fraction of tuning goes as $\frac{1}{M_{\text {susy }}^{4}}$. Because the growth in the number of "almost supersymmetric" vacua at small $\Lambda$ is only logarithmic in $M_{\text {susy }}$, it is not enough to overcome the power-law suppression arising from the necessity of tuning the Higgs.

In the subset of vacua where $R$-symmetry is unbroken, there may be even further enhancements associated with the phenomenological condition of a small cosmological constant. For the "almost supersymmetric" ensemble, the vanishing of the tree-level cosmological constant is equivalent to the vanishing of the tree-level superpotential, $W_{0}=0 . R$-symmetry is a discrete symmetry which would guarantee this (as the superpotential is charged under $R$-symmetry). But in the "almost supersymmetric" ensemble, supersymmetry can still be broken dynamically at a scale $M_{\text {susy }}$, which may also be associated with dynamical $R$ symmetry breaking. In this case one would naturally expect the relations

$$
|D W|^{2} \sim M_{\text {susy }}^{4} \quad 3 \frac{|W|^{2}}{M_{p}^{2}} \sim m_{3 / 2}^{2} M_{p}^{2},
$$

where $m_{3 / 2}$ is the gravitino mass. If $M_{\text {susy }}^{2} \sim m_{3 / 2} M_{p}$, then these terms are of the same order. Note that this is precisely the case of intermediate scale supersymmetry breaking, which is 
what would arise if the dominant transmission mechanism of supersymmetry breaking to the Standard Model sector is gravity. In this case, we find that the fine-tuning required to fix an appropriately small cosmological constant is only $\frac{\Lambda}{M_{i n t}^{4}}$. It can never be smaller than this (due to the relationship between $|W|$ and the gravitino mass). But for a larger supersymmetry breaking scale, more fine-tuning $\left(\frac{\Lambda}{M_{\text {susy }}^{4}}\right)$ would be required.

Note of course that in models such as this, the enhancement is greater for smaller values of the gravitino mass. As such, there are many more vacua in this class (tree-level $R$-symmetric) with extremely small gravitino mass than there are with phenomenologically viable gravitino mass. But this should not bother us terribly. We have already conceded that our universe is highly non-generic, and we are not attempting to find a selection principle which selects an allowed value of the gravitino mass. The name of the game is to consider gravitino masses which are allowed by experiment, and see if we can estimate the number of models obtainable by various constructions which are consistent with this constraint. We find that in constructions with tree-level supersymmetry breaking, most viable models have high-scale SUSY-breaking. For models in the "almost supersymmetric" ensemble, most viable vacua have intermediate scale supersymmetry-breaking. This fraction is even larger among the "almost supersymmetric" ensemble vacua for which there is a tree-level $R$-symmetry.

One can then ask how large is the suppression required to obtain vacua with unbroken tree-level $R$-symmetry. Dine et al. [53] have studied algebraic constructions of Calabi-Yau manifolds, and found in all cases they studied that discrete symmetries remained unbroken when at most one-third of fluxes were turned on. This is quite significant, because it implies a suppression factor of order $\left(L_{*}\right)^{\frac{2}{3} n}$, where $n$ is the number of complex structure moduli (and $4 n+4$ is the overall number of fluxes). Dewolfe 54 further argues for the suppression of the number of vacua with $W=0$ (required for unbroken $R$-symmetry) in a large algebraic class, though the ratio of exponents may be subleading in $n$. For sufficiently large $L_{*}$ and $n$, the suppression required to obtain tree-level $R$-symmetry outweighs the enhancement in the fraction of $R$-symmetric vacua with viable Higgs mass and cosmological constant.

Thus far in the analysis, we have assumed a relatively flat distribution for the complex structure moduli and axio-dilaton. In fact, the distribution of these moduli is determined by the curvature of the moduli space, and can be concentrated at singularities, such as the conifold. As was shown in KKLT, if supersymmetry is broken by adding anti-D3-branes, then they are naturally pushed to the end of the warped throat, generating an exponentially small SUSY-breaking term in the potential. This biasing of the distribution acts in favor of providing more vacua with low-energy supersymmetry breaking. 
Note that we have restricted ourselves to the class of models which retain supersymmetry at the Kaluza-Klein scale and the string scale. One expects that there are solutions where supersymmetry is broken even at the KK or string scale [13, 14, and there may be many of them. It would be interesting to compare the distribution of the supersymmetry breaking scale in the larger ensemble (where supersymmetry above these higher scales is not assumed) to the ensembles we have already considered (which themselves include branches where highscale supersymmetry breaking is dominant).

\section{Distributions of Standard Model-like Vacua}

We have already discussed flux vacua distributions under the phenomenological constraints of small cosmological constant and reasonable Higgs mass. The next constraint to impose would be to demand the appearance of Standard Model gauge group and matter content. This constraint has been studied in the Type IIB context [55, 56, 57, 58, 59].

In the context of Type IIB string theory, the simplest way to arrive at the SM gauge group and matter content would be from the open string sector arising from intersecting D-branes. However, these space-filling D-branes contribute to the RR-tadpole constraints, just as the orientifold planes do. Any excess negative D3-brane charge can be canceled off by the charge induced from RR and NSNS-fluxes. But these fluxes also serve to generate a tree-level GVW superpotential which can fix the complex structure moduli. In this way, the intersecting brane world story is joined to our phenomenological study of the landscape 60, 61]. We briefly review this construction here.

Our starting point is again Type IIB string theory compactified on an orientifolded Calabi-Yau 3-fold. The simplest and most studied case is actually an orbifold, $T^{6} / Z_{2} \times Z_{2}$. But these considerations apply more generally. The orientifold action leaves us with some set of orientifold planes which contribute to the RR-tadpoles. Their contributions must all be canceled as before by some combination of branes and flux-induced charge. Generally we have several RR-tadpole conditions to satisfy. We can do so with a variety of branes, and the gauge theory arising from these branes should include a sector which carries Standard Model gauge group and matter content. 


\subsection{Standard Model constructions}

The gauge group and matter content of the branes depend on the type of orientifold we study and the choice of cycles on which we wrap the D-branes. For concreteness and simplicity, we choose the orientifold of $T^{6} / Z_{2} \times Z_{2}$ (this has also been studied in [62, 63]). We then only have O3- and O7-planes, as well as 3 Kähler moduli and 51 complex structure moduli. We will add so-called "magnetized" D-branes [64, in which the gauge theory on the D-brane has magnetic fields turned on, inducing lower brane charge as well. The orientifold action ensures that the D5-brane and D9-brane charge of any magnetized brane cancels against the charge of the orientifold image. As a result, we need not worry about the corresponding RR-tadpole conditions. ${ }^{14}$ But the total D3-brane and D7-brane charges carried by these magnetized branes must cancel against the charges of the orientifold planes and fluxes.

The gauge group and matter content of any set of branes is basically determined by the cycles which they wrap, and the topological intersection numbers between these cycles. We consider the case where all branes are fixed either at orbifold or orientifold planes (this ensures that we have an odd number of generations[55]). If a stack of $N$ branes lies at an orbifold fixed point, the corresponding gauge group is $U\left(\frac{N}{2}\right)$, while if they lie at orientifold fixed plane the gauge group is $U S p(N)$. In either case, $N$ must be even.

The chiral matter content is determined by the topological intersection number $I_{a b}$ between the branes. ${ }^{15}$ If two branes $a$ and $b$ intersect, then there are $I_{a b}$ chiral multiplets transforming in the bifundamental $\left(G_{a}, \bar{G}_{b}\right)$. If $b^{\prime}$ represents the orientifold image of $b$, then we also find $I_{a b^{\prime}}$ chiral multiplets transforming in the $\left(G_{a}, G_{b}\right)$. If a brane intersects its own orientifold image, we have additional chiral multiplets transforming in the symmetric and anti-symmetric representation of the appropriate gauge group.

The idea is to find two sets of branes, which we call "visible sector branes" and "hidden sector branes." The visible sector branes contain the gauge group of the Standard Model and it's chiral matter content, while the hidden sector branes ensure that the D7-brane RR tadpole conditions are satisfied. ${ }^{16}$ The D3-brane RR tadpole condition must be undersaturated, with the difference being made up by charge induced from the RR and NSNS 3-form

\footnotetext{
${ }^{14}$ There is a subtlety here. There is in fact a remaining $\mathrm{K}$-theoretic $Z_{2}$ tadpole constraint, which one must be sure to satisfy 65, 66, 67.

${ }^{15}$ It is often easier to think of these branes in terms of a mirror picture, where they appear as D6-branes wrapping special Lagrange 3-cycles, with no magnetic fields. $I_{a b}$ is then simply the oriented intersection number. When we think of a CY mirror in this context, we are first setting the fluxes to zero. This is justified for our purpose here, as it does not affect the open string theory gauge group or matter content.

${ }^{16}$ There may be chiral matter which is charged under both visible sector brane gauge groups and hidden sector brane gauge groups. This matter will be referred to as exotic.
} 
fluxes. As we have seen, the number of flux vacua increases with the amount of flux which we can turn on.

But as before, we wish to find constructions which are supersymmetric (so that we can later add in lower scale supersymmetry-breaking by hand). So if we add several stacks of branes, we wish to be sure that they respect the same supersymmetries 68. This condition is most easily understood from the the dual IIA picture, in which we have D6-branes with no magnetic field. In this case, as shown in [69], the condition for two branes to preserve the same supersymmetry is that they are related by a rotation of $0 \bmod 2 \pi$ (this is equivalent to the vanishing of the NSNS tadpole constraint). Note however, that violation of this condition does not show that supersymmetry is broken; it merely shows that a Fayet-Iliopoulos term is turned on. Roughly, we have $|\delta \theta| \sim\left|\xi_{F I}\right|$. For each brane (indexed by $j$ ), we have ${ }^{17}$ a $D$-term potential[70]

$$
V_{D}^{j}=\left(\sum_{i} q_{i}^{j}\left|\phi_{i}^{j}\right|^{2}-\xi^{j}\right)^{2}
$$

where the scalars $\phi_{i}^{j}$ arise from open strings stretching between branes $i$ and $j$, and $q_{i}^{j}$ are the scalar charges under the $U(1)$ of brane $j$. We see that if the NSNS tadpole constraints are satisfied, then $\xi^{j}=0$ and the $D$-term potential will vanish if we also set $\phi_{i}^{j}=0$. However, even if some of the $\xi$ 's are non-zero, some of the open string scalars $\phi$ will become tachyonic if their charge $q$ has the appropriate sign. In this case, the scalars can get vevs which cancel the FI-term and set the $D$-term to zero. This process is known as brane recombination; the veving of the tachyonic open-string scalar $\phi_{i}^{j}$ corresponds to the branes $i$ and $j$ forming a bound state.

Bearing in mind that this brane recombination process can occur, we find that it is not necessary for the NSNS tadpole conditions to be satisfied in order to maintain supersymmetry. Indeed, if we have $r$ branes the we have $r D$-terms, but we generically have $\sim r^{2}$ open string scalars (since our branes generically have non-zero topological intersection with one another). In this case, we generically find for any brane a scalar charged with either sign of charge. In such a case, we generally can restore supersymmetry by some type of brane recombination for any values of the FI-terms.

\subsection{Some results}

One particular construction of visible and hidden sector branes was found by Marchesano and Shiu [57, 58], and generated a visible sector yielding an $U(4) \times S U(2)_{L} \times S U(2)_{R}$ extension

\footnotetext{
${ }^{17}$ There is no summation on $j$.
} 
of the Standard Model. They searched for models in which all of the FI-terms vanished, thus avoiding some of the complications of brane recombination. Their model is described by the brane embedding

$$
\begin{array}{cc}
N_{a}=6 & (1,0)(3,1)(3,-1) \\
N_{b}=2 & (0,1)(1,0)(0,-1) \\
N_{c}=2 & (0,1)(0,-1)(1,0) \\
N_{d}=2 & (1,0)(3,1)(3,-1) \\
N_{h 1}=2 & (-2,1)(-3,1)(-4,1) \\
N_{h 2}=2 & (-2,1)(-4,1)(-3,1) \\
N_{h 3}=8 & (1,0)(1,0)(1,0)
\end{array}
$$

where the wrapping numbers $(n, m)$ describe the integer quantized magnetic flux and winding, respectively, of the brane on each of the three tori. The first 4 brane stacks are the visible sector and the last three brane stacks are the hidden sector. This model allows one to obtain one quantized unit of D3-brane charge from fluxes (which thus fix the complex structure moduli and axio-dilaton). Indeed, it was shown in [71] that this is in fact the only model on this orientifold with the given choice of visible sector, non-zero flux and vanishing FI-terms.

However, several choices of hidden sector can be exhibited with allow much larger amounts of flux, once brane recombination is utilized (i.e., we drop the demand for vanishing FIterms). Several of these models where exhibited[71, 72], the largest of which exhibited $N=9$ units of quantized D3-brane charge induced from the fluxes. The counting arguments we discussed before suggest that we would find $\sim 10^{30}$ flux vacua in this model with the chosen visible and hidden sector brane content.

There are in fact several other choices of visible sector which produce different extensions of the Standard Model, and they can be studied in a similar vein [56].

\subsection{Standard Model phenomenology considerations}

We see that we can potentially find large numbers of flux vacua with the Standard Model gauge group and chiral matter content. One can then study the distribution of parameters such as the tree-level cosmological constant for these models as reviewed earlier. The hope is to utilize the large number of vacua to estimate which fraction of these vacua happen to have low-energy parameters which are within experimental precision of the real world. 
For example, Douglas [30] estimates that a fine-tuning of parameters on the order of $10^{238}$ is need to "get the numbers right," i.e., to fine-tune the actual masses and couplings (plus cosmological constant) from their natural values to experimentally measured values with current precision. Thus, if we find a number of vacua $\mathcal{N}$ with the right SM matter content such that $\mathcal{N} \gg 10^{238}$, and the distribution of scalar vev's is fairly uniform, then we may be quite confident that there exists a string vacua whose low-energy parameters match the real world to within experimental precision. We are also in a position to study the phenomenology of this set of vacua.

An extended discussion of this phenomenology is beyond the scope of this review [71], but there are a few points worth noting. Our initial paradigm was to fix the moduli at a scale well above the supersymmetry breaking scale, in order to ensure that non-supersymmetric corrections to the potential did not destabilize the solution. But we do not include here the open string scalars which are charged under the Standard Model. These scalars must arise from open string non-vector-like chiral multiplets, and the scalars for these multiplets cannot get mass except at the SUSY-breaking scale. These scalars are coupled to the Kähler moduli through the $D$-term equations. If the Kähler moduli are not fixed by $F$-terms, then they might be fixed by D-terms instead, but this would be at the SUSY-breaking scale. This could be problematic for the stability of the solution. Alternatively, if the Kähler moduli are fixed by $F$-terms, then the open string scalars would likely break supersymmetry by a combination of $F$-terms and $D$-terms. Interesting features of supersymmetry breaking in this context are also discussed in [73, 29].

Ubiquitous features of these types of constructions are non-chiral exotics, extra $U(1)$ 's and potential discrete symmetries. We can study the distribution of these properties among the class of vacua which admit the Standard Model. This counting can thus be convolved with the usual phenomenological discussion of these features.

\section{Discussion - an Infinity of Vacua}

We have seen that there are many very concrete calculations we can perform, from which we can derive the distribution of low-energy properties among flux vacua in a variety of specific constructions. The question is, "how can we use these distributions to make contact with phenomenology?" Unfortunately, our limited understanding of non-supersymmetric string compactifications makes it difficult for us to make progress. But we have already seen that the study of these distributions does provide us clues which can direct further 
phenomenological work. In addition, we can also study some formal string theory questions which have significant implications for the role of string theory in phenomenology.

One of the questions we hope to better answer with this study of the landscape is how many string vacua match the real world to experimental precision, and in particular, is the number finite or infinite [4]. One may be tempted already to speculate on the implications of the answer to this question 30 .

It is sometimes treated as disastrous for the predictivity of string theory if the number of phenomenologically viable vacua is infinite. However, that is not necessarily the case. Even if the number of viable vacua is infinite, it can still be possible to make predictions regarding the values of masses and low-energy couplings (in principle). Consider the parameter space of a low-energy description of string theory vacua. This can be thought of as the parameter space of the low-energy effective field theory, and is consequently infinite dimensional (as the number of possible couplings in effective field theory is infinite). The real world, to within experimental uncertainty, occupies some ball ${ }^{18}$ in this space. As experimental data becomes more precise, the size of the ball shrinks. The various consistent vacua of string theory appear as points (not necessarily isolated) in this space. If the ball shrinks to a size such that there is exactly one string vacuum inside the ball, then string theory gives a unique prediction for which vacuum we live in and what its low-energy properties are (to arbitrary accuracy). Even if there are an infinite number of isolated flux vacua, this will always occur in principle as long as the set of string vacua do not have limit points in the ball.

We may have a situation in which, for any open set in parameter space containing the theory which exactly describes the real world, there exists at least one string vacuum within that open set. Even in this case, it is still possible in principle for string theory to make useful predictions of low-energy couplings. For example, suppose we are only interested in two parameters: the electron mass and the proton mass. It may be that the infinite number of phenomenologically viable vacua are effectively confined to a 1-dimensional surface in this two-dimensional parameter subspace. This means that we would not be able to predict either the electron mass or the proton mass individually. But if new experiments gave increased precision in the measurement of the electron mass, this would translate into a prediction for the next order of precision in the proton mass.

Of course, it is also possible that the set of vacua densely fills the space of low-energy parameters. ${ }^{19}$ In this case, it is true that string theory is unable to predict any low-energy

\footnotetext{
${ }^{18}$ By "ball," we simply mean an open set. It can of course have a highly complicated shape, depending on how the space is parameterized.

${ }^{19}$ For some contrary arguments, see [7].
} 
parameters, or find any correlations between them. It is interesting to note the implications of this result for the Bekenstein bound. The Bekenstein bound [76, 77] on the entropy of a system is given by

$$
S \leq \frac{2 \pi E R}{\hbar c}
$$

One may show that this bound can be trivially violated by field-theoretic systems (for example, a very large number of scalars very weakly coupled to gravity[78]). But one may be suspicious of these counter-examples, because there is no known realization of them in a consistent theory of quantum gravity, such as string theory. If it were in fact true, however, that the set of string vacua densely fills the space of all possible low-energy effective field theories, then these counter-examples would be legitimate and the Bekenstein bound would fail. One may view this as a way for the string landscape to provide evidence for or against the Bekenstein bound. Alternatively, one may view the circumstantial evidence in favor of the Bekenstein bound as evidence for the claim that string vacua do not densely pack the space of possible low-energy effective field theories.

This question addresses the formal predictivity of string theory. But a related, and arguably more relevant question is whether experimental precision can ever give us constraints tight enough (that is, a small enough "ball") to allow string theory to make useful predictions. Alternatively, one might ask if string theory could exclude, as a practical matter, any features which model-builders find interesting. It may be easier for string theory to provide input to model-building by determining that particular classes of constructions are unlikely (or quite likely) to yield vacua exhibiting particular interesting features. Current studies of distributions on the landscape already give hints as to how this program can proceed.

These are just a few examples of the variety of questions, both formal and phenomenological, which we can study via the landscape. Clearly, there is much more work which can be done is this emerging field. The most exciting results and prospects appear to lie in the future.

\section{Acknowledgements}

We gratefully acknowledge T. Banks, F. Denef, M. Dine, M. Douglas, S. Kachru, F. Larsen, S. Sethi, G. Shiu and L. Susskind for useful discussions. We are especially grateful to J. Wells for discussions and collaboration at all stages of our study of this field. This work is supported in part by NSF grant PHY-0314712. 


\section{References}

[1] S. Kachru, R. Kallosh, A. Linde and S. P. Trivedi, Phys. Rev. D 68, 046005 (2003) hep-th/0301240.

[2] B. S. Acharya, arXiv:hep-th/0212294.

[3] J. P. Derendinger, C. Kounnas, P. M. Petropoulos and F. Zwirner, Nucl. Phys. B 715, 211 (2005) hep-th/0411276. G. Villadoro and F. Zwirner, hep-th/0503169. J. P. Derendinger, C. Kounnas, P. M. Petropoulos and F. Zwirner, hep-th/0503229.

[4] O. DeWolfe, A. Giryavets, S. Kachru and W. Taylor, hep-th/0505160. P. G. Camara, A. Font and L. E. Ibanez, hep-th/0506066.

[5] A. Strominger, Nucl. Phys. B 274, 253 (1986). J. Polchinski and A. Strominger, Phys. Lett. B 388, 736 (1996) hep-th/9510227. K. Becker and M. Becker, Nucl. Phys. B 477, 155 (1996) hep-th/9605053. J. Michelson, Nucl. Phys. B 495, 127 (1997) hep-th/9610151. M. Grana, Phys. Rept. 423, 91 (2006) hep-th/0509003.

[6] T. R. Taylor and C. Vafa, Phys. Lett. B 474, 130 (2000) hep-th/9912152. B. R. Greene, K. Schalm and G. Shiu, Nucl. Phys. B 584, 480 (2000) hep-th/0004103. G. Curio, A. Klemm, D. Lust and S. Theisen, Nucl. Phys. B 609, 3 (2001) hep-th/0012213.

[7] S. Sethi, C. Vafa and E. Witten, Nucl. Phys. B 480, 213 (1996) hep-th/9606122. K. Dasgupta, G. Rajesh and S. Sethi, JHEP 9908, 023 (1999) hep-th/9908088. S. Kachru, M. B. Schulz and S. Trivedi, JHEP 0310, 007 (2003) hep-th/0201028. S. Hellerman, J. McGreevy and B. Williams, JHEP 0401, 024 (2004) hep-th/0208174. A. Dabholkar and C. Hull, JHEP 0309, 054 (2003) hep-th/0210209. P. K. Tripathy and S. P. Trivedi, JHEP 0303, 028 (2003) hep-th/0301139. P. S. Aspinwall and R. Kallosh, JHEP 0510, 001 (2005) hep-th/0506014. K. Behrndt, M. Cvetic and T. Liu, hep-th/0512032.

[8] S. B. Giddings, S. Kachru and J. Polchinski, Phys. Rev. D 66, 106006 (2002) hep-th/0105097.

[9] R. Bousso and J. Polchinski, JHEP 0006, 006 (2000) hep-th/0004134.

[10] A. Giryavets, S. Kachru, P. K. Tripathy and S. P. Trivedi, JHEP 0404, 003 (2004) hep-th/0312104. A. Giryavets, S. Kachru and P. K. Tripathy, JHEP 0408, 002 (2004) hep-th/0404243. L. Gorlich, S. Kachru, P. K. Tripathy and S. P. Trivedi, 
hep-th/0407130. O. DeWolfe, A. Giryavets, S. Kachru and W. Taylor, JHEP 0502, 037 (2005) hep-th/0411061. I. Antoniadis and T. Maillard, Nucl. Phys. B 716, 3 (2005) hep-th/0412008. I. Antoniadis, A. Kumar and T. Maillard, hep-th/0505260. E. Bergshoeff, R. Kallosh, A. K. Kashani-Poor, D. Sorokin and A. Tomasiello, JHEP 0510, 102 (2005) hep-th/0507069. J. J. Blanco-Pillado, R. Kallosh and A. Linde, arXiv:hep-th/0511042.

[11] F. Denef, M. R. Douglas and B. Florea, JHEP 0406, 034 (2004) hep-th/0404257.

[12] F. Denef, M. R. Douglas, B. Florea, A. Grassi and S. Kachru, hep-th/0503124.

[13] A. Maloney, E. Silverstein and A. Strominger, hep-th/0205316. E. Silverstein, hep-th/0106209.

[14] A. Saltman and E. Silverstein, hep-th/0411271.

[15] M. R. Douglas, Comptes Rendus Physique 5, 965 (2004) hep-th/0409207. N. ArkaniHamed, Talk at Workshop on String Phenomenology, Perimeter Institute, March 28April 1, 2005.

[16] H. Firouzjahi, S. Sarangi and S. H. H. Tye, JHEP 0409, 060 (2004) hep-th/0406107. A. Kobakhidze and L. Mersini-Houghton, hep-th/0410213 L. Mersini-Houghton, Class. Quant. Grav. 22, 3481 (2005) hep-th/0504026. S. Sarangi and S. H. Tye, hep-th/0505104. R. Holman and L. Mersini-Houghton, hep-th/0511102, R. Holman and L. Mersini-Houghton, hep-th/0511112. R. Brustein and S. P. de Alwis, hep-th/0511093. R. Holman and L. Mersini-Houghton, hep-th/0512070. L. MersiniHoughton, hep-th/0512304.

[17] T. Banks, hep-th/0412129.

[18] T. Banks, M. Dine and E. Gorbatov, JHEP 0408, 058 (2004) hep-th/0309170. B. Freivogel and L. Susskind, Phys. Rev. D 70, 126007 (2004) hep-th/0408133.

[19] E. Farhi and A. H. Guth, Phys. Lett. B 183, 149 (1987).

[20] B. Freivogel, V. E. Hubeny, A. Maloney, R. Myers, M. Rangamani and S. Shenker, hep-th/0510046.

[21] G. L. Alberghi, D. A. Lowe and M. Trodden, JHEP 9907, 020 (1999) arXiv:hep-th/9906047. 
[22] E. Silverstein, hep-th/0407202, A. Misra and A. Nanda, Fortsch. Phys. 53, 246 (2005) hep-th/0407252. K. R. Dienes, E. Dudas and T. Gherghetta, hep-th/0412185.

[23] N. Arkani-Hamed, S. Dimopoulos and S. Kachru, hep-th/0501082.

[24] S. Kachru and A. K. Kashani-Poor, JHEP 0503, 066 (2005) hep-th/0411279.

[25] M. Dine and N. Seiberg, Phys. Lett. B 162, 299 (1985).

[26] S. Kachru, J. Pearson and H. Verlinde, JHEP 0206, 021 (2002) hep-th/0112197.

[27] S. P. de Alwis, hep-th/0506266.

[28] S. J. Gates, M. T. Grisaru, M. Rocek and W. Siegel, Front. Phys. 58, 1 (1983) hep-th/0108200.

[29] K. Choi, A. Falkowski, H. P. Nilles, M. Olechowski and S. Pokorski, JHEP 0411, 076 (2004) hep-th/0411066.

[30] M. R. Douglas, JHEP 0305, 046 (2003) hep-th/0303194.

[31] S. Ashok and M. R. Douglas, JHEP 0401, 060 (2004) hep-th/0307049.

[32] F. Denef and M. R. Douglas, JHEP 0405, 072 (2004) hep-th/0404116.

[33] S. Gukov, C. Vafa and E. Witten, Nucl. Phys. B 584, 69 (2000) [Erratum-ibid. B 608, 477 (2001)] hep-th/9906070.

[34] K. Becker, M. Becker, M. Haack and J. Louis, JHEP 0206, 060 (2002) hep-th/0204254. G. von Gersdorff and A. Hebecker, Phys. Lett. B 624, 270 (2005) hep-th/0507131. M. Berg, M. Haack and B. Kors, hep-th/0508043. M. Berg, M. Haack and B. Kors, hep-th/0508171.

[35] A. E. Nelson and N. Seiberg, Nucl. Phys. B 416, 46 (1994) arXiv:hep-ph/9309299.

[36] D. Robbins and S. Sethi, Phys. Rev. D 71, 046008 (2005) hep-th/0405011.

[37] R. Brustein and S. P. de Alwis, Phys. Rev. D 69, 126006 (2004) hep-th/0402088.

[38] A. Saltman and E. Silverstein, JHEP 0411, 066 (2004) hep-th/0402135.

[39] I. R. Klebanov and M. J. Strassler, JHEP 0008, 052 (2000) hep-th/0007191.

[40] G. W. Moore, arXiv:hep-th/0401049, R. Kallosh, arXiv:hep-th/0509112, A. Giryavets, arXiv:hep-th/0511215. 
[41] S. P. de Alwis, Phys. Lett. B 628, 183 (2005) hep-th/0506267.

[42] H. X. Yang, hep-th/0511030.

[43] S. Kachru, R. Kallosh, A. Linde, J. Maldacena, L. McAllister and S. P. Trivedi, JCAP 0310, 013 (2003) hep-th/0308055.

[44] M. Kontsevich, "Homological algebra of mirror symmetry," Proceedings of ICM (Zürich, 1994),120-139. Birkhäuser, Basel, 1995, alg-geom/9411018. I. Smith, R. P. Thomas, S.-T. Yau, "Symplectic conifold transitions," Jour. Diff. Geom. 62, 209 (2002) math.SG/0209319. I. Smith, R. P. Thomas "Symplectic surgeries from singularities," Turkish J. Math. 27, 231 (2003) math.SG/0212213. N. Hitchen, Quart. J. Math. Oxford Ser. 54, 281 (2003) math.dg/0209099. M. Grana, R. Minasian, M. Petrini and A. Tomasiello, "Supersymmetric backgrounds from generalized Calabi-Yau manifolds," JHEP 0408 (2004) 046 hep-th/0406137.

[45] B. S. Acharya, F. Denef and R. Valandro, hep-th/0502060.

[46] J. P. Conlon and F. Quevedo, JHEP 0410, 039 (2004) hep-th/0409215. J. Kumar and J. D. Wells, Phys. Rev. D 71, 026009 (2005) hep-th/0409218.

[47] J. Gomis, F. Marchesano and D. Mateos, JHEP 0511, 021 (2005) hep-th/0506179.

[48] J. Distler and U. Varadarajan, hep-th/0507090.

[49] S. Weinberg, Phys. Rev. Lett. 59, 2607 (1987).

[50] L. Susskind, hep-th/0302219. M. R. Douglas, hep-th/0405279. L. Susskind, hep-th/0405189.

[51] F. Denef and M. R. Douglas, JHEP 0503, 061 (2005) hep-th/0411183. M. Dine, D. O’Neil and Z. Sun, JHEP 0507, 014 (2005) hep-th/0501214.

[52] M. Dine, E. Gorbatov and S. Thomas, hep-th/0407043.

[53] M. Dine and Z. Sun, hep-th/0506246.

[54] O. DeWolfe, hep-th/0506245.

[55] R. Blumenhagen, L. Goerlich, B. Kors and D. Lust, JHEP 0010, 006 (2000) hep-th/0007024. C. Angelantonj, I. Antoniadis, E. Dudas and A. Sagnotti, Phys. Lett. B 489, 223 (2000) hep-th/0007090. G. Aldazabal, S. Franco, L. E. Ibanez, R. Rabadan 
and A. M. Uranga, JHEP 0102, 047 (2001) hep-ph/0011132. G. Aldazabal, S. Franco, L. E. Ibanez, R. Rabadan and A. M. Uranga, J. Math. Phys. 42, 3103 (2001) hep-th/0011073. D. Cremades, L. E. Ibanez and F. Marchesano, JHEP 0207, 009 (2002) hep-th/0201205. R. Blumenhagen, V. Braun, B. Kors and D. Lust, hep-th/0210083. A. M. Uranga, Class. Quant. Grav. 20, S373 (2003) hep-th/0301032. D. Lust, Class. Quant. Grav. 21, S1399 (2004) hep-th/0401156. E. Kiritsis, Fortsch. Phys. 52, 200 (2004) hep-th/0310001. G. Honecker and T. Ott, Phys. Rev. D 70, 126010 (2004) [Erratum-ibid. D 71, 069902 (2005)] hep-th/0404055. C. Kokorelis, hep-th/0410134.

[56] M. Cvetic, T. Li and T. Liu, hep-th/0501041.

[57] F. Marchesano and G. Shiu, Phys. Rev. D 71, 011701 (2005) hep-th/0408059.

[58] F. Marchesano and G. Shiu, JHEP 0411, 041 (2004) hep-th/0409132.

[59] F. Gmeiner, R. Blumenhagen, G. Honecker, D. Lust and T. Weigand, hep-th/0510170.

[60] T. P. T. Dijkstra, L. R. Huiszoon and A. N. Schellekens, Nucl. Phys. B 710, 3 (2005) hep-th/0411129; Phys. Lett. B 609, 408 (2005) hep-th/0403196.

[61] R. Blumenhagen, F. Gmeiner, G. Honecker, D. Lust and T. Weigand, Nucl. Phys. B 713, 83 (2005) hep-th/0411173.

[62] R. Gopakumar and S. Mukhi, Nucl. Phys. B 479, 260 (1996) hep-th/9607057.

[63] S. Forste, G. Honecker and R. Schreyer, Nucl. Phys. B 593, 127 (2001) hep-th/0008250. M. Cvetic, G. Shiu and A. M. Uranga, Phys. Rev. Lett. 87, 201801 (2001) hep-th/0107143. M. Cvetic, G. Shiu and A. M. Uranga, Nucl. Phys. B 615, 3 (2001) hep-th/0107166. M. Cvetic, I. Papadimitriou and G. Shiu, Nucl. Phys. B 659, 193 (2003) [Erratum-ibid. B 696, 298 (2004)] hep-th/0212177. M. Cvetic and I. Papadimitriou, Phys. Rev. D 67, 126006 (2003) hep-th/0303197. M. Cvetic, T. Li and T. Liu, Nucl. Phys. B 698, 163 (2004) hep-th/0403061. M. Cvetic, P. Langacker, T. j. Li and T. Liu, Nucl. Phys. B 709, 241 (2005) hep-th/0407178.

[64] J. F. G. Cascales and A. M. Uranga, JHEP 0305, 011 (2003) hep-th/0303024.

[65] A. M. Uranga, Nucl. Phys. B 598, 225 (2001) hep-th/0011048.

[66] E. Witten, Phys. Lett. B 117, 324 (1982).

[67] E. Witten, JHEP 9812, 019 (1998) hep-th/9810188. 
[68] N. Ohta and P. K. Townsend, Phys. Lett. B 418, 77 (1998) hep-th/9710129.

[69] M. Berkooz, M. R. Douglas and R. G. Leigh, Nucl. Phys. B 480, 265 (1996) hep-th/9606139.

[70] S. Kachru and J. McGreevy, Phys. Rev. D 61, 026001 (2000) hep-th/9908135.

[71] J. Kumar and J. D. Wells, JHEP 0509, 067 (2005) hep-th/0506252.

[72] For a listing of solutions consistent with all constraints, please see http://www.umich.edu/ jwells/hiddensectors.txt

[73] C. P. Burgess, R. Kallosh and F. Quevedo, JHEP 0310, 056 (2003) hep-th/0309187.

D. Lust, S. Reffert and S. Stieberger, Nucl. Phys. B 706, 3 (2005) hep-th/0406092;

P. G. Camara, L. E. Ibanez and A. M. Uranga, Nucl. Phys. B 708, 268 (2005) hep-th/0408036. A. Font and L. E. Ibanez, JHEP 0503, 040 (2005) hep-th/0412150. K. Choi, A. Falkowski, H. P. Nilles and M. Olechowski, Nucl. Phys. B 718, 113 (2005) hep-th/0503216 J. P. Conlon, F. Quevedo and K. Suruliz, hep-th/0505076. D. E. Diaconescu, B. Florea, S. Kachru and P. Svrcek, hep-th/0512170. J. P. Derendinger, C. Kounnas and P. M. Petropoulos, hep-th/0601005.

[74] M. R. Douglas and Z. Lu, arXiv:hep-th/0509224.

[75] C. Vafa, hep-th/0509212. N. Arkani-Hamed, L. Motl, A. Nicolis and C. Vafa, hep-th/0601001.

[76] J. D. Bekenstein, Phys. Rev. D 23, 287 (1981).

[77] J. D. Bekenstein, Phys. Rev. D 9, 3292 (1974).

[78] D. N. Page, Phys. Rev. D 26, 947 (1982). 